\title{
Interfacial Electronic Properties Dictate Li Dendrite Growth in Solid Electrolytes
}

\author{
Hong-Kang $\operatorname{Tian}^{1 *}$, Zhe Liu ${ }^{2 *}$, Yanzhou $\mathrm{Ji}^{2}$, Long-Qing Chen ${ }^{2 \#}$, Yue Qi ${ }^{1 \#}$ \\ ${ }^{1}$ Department of Chemical Engineering and Materials Science, Michigan State University, East \\ Lansing, MI 48824, USA \\ ${ }^{2}$ Department of Materials Science and Engineering and the Materials Research Institute, The \\ Pennsylvania State University, University Park, PA 16802, USA
}

\section{DFT Calculation methodology}

\section{I.1 Atomic structures}

\section{Cubic Li7 $\mathrm{La}_{3} \mathrm{Zr}_{2} \mathrm{O}_{12}(\mathrm{c}-\mathrm{LLZO})$}

It was reported that $\mathrm{Al}$ doping would help to maintain the cubic structure and achieve higher $\mathrm{Li}$ conductivity, such as $\mathrm{Li}_{6.24} \mathrm{Al}_{0.24} \mathrm{La}_{3} \mathrm{Zr}_{2} \mathrm{O}_{11.98}$ [1]. Considering the low concentration of $\mathrm{Al}$, a cubic $\mathrm{Li}_{7} \mathrm{La}_{3} \mathrm{Zr}_{2} \mathrm{O}_{12}$ as characterized by Xie et al. [2] was used in our model to represent c-LLZO, which contains eight formula units and 192 atoms. The Li atoms were assigned on the partially occupied 24d and 96h sites in c-LLZO via the distribution principle proposed by Cussen and O'Callaghan $[3,4]$ and Xie et al. [2], which minimizes the energy by avoiding the electrostatically-unfavorable Li configurations. The bulk structure was obtained from our previous study [5] that has the lowest energy among different distributions of Li atoms. The surface structure of c-LLZO was also obtained from previous studies [5], which suggested the orientation of (110) and the termination of Li-La atoms with a stoichiometric ratio is the most energetic-favorable one.

\section{$\beta-\mathrm{Li}_{3} \mathrm{PS}_{4}$}

The Li distribution in bulk $\beta-\mathrm{Li}_{3} \mathrm{PS}_{4}$ was measured in another study [6] by x-ray diffraction that showed the 8d site (Wyckoff notation) was fully occupied, 4b and 4c site was 70\% and 30\% occupied by Li atoms, respectively. On the other hand, a DFT study [7] for different configurations 
of $\mathrm{Li}$ atoms in bulk $\beta-\mathrm{Li}_{3} \mathrm{PS}_{4}$ revealed that the structure with $8 \mathrm{~d}$ and $4 \mathrm{~d}$ fully occupied but $4 \mathrm{c}$ empty has the lowest energy. In this study, we chose the atomic structure that was suggested by the abovementioned computational research, which also simplifies the configuration of Li atoms. A bulk $\beta-\mathrm{Li}_{3} \mathrm{PS}_{4}$ unit cell consists of 16 atoms with a space group of Pnma. Considering the $\mathrm{PS}_{4}$ polyhedral blocks in $\beta-\mathrm{Li}_{3} \mathrm{PS}_{4}$, a suitable surface orientation would be the one that preserves the $\mathrm{PS}_{4}$ units and Li-S bonds, which we found that the (100) direction with symmetrical termination of $\mathrm{S}$ atoms and a stoichiometric ratio matches the requirements. Upon calculating the surface energy, which converged with six or more layers of $\mathrm{PS}_{4}$, it is as low as $0.19 \mathrm{~J} / \mathrm{m}^{2}$ that indicates this is an energetic-favorable slab.

\section{LATP}

It was reported that the ionic conductivity of $\mathrm{Li}_{1+\mathrm{x}} \mathrm{Al}_{\mathrm{x}} \mathrm{Ti}_{2-\mathrm{x}}\left(\mathrm{PO}_{4}\right)_{3}$ reaches the maximum at $\mathrm{x}=0.3$ [8], and the crystal structure and the atom positions have been revealed by a neutron diffraction analysis $[9,10]$. The unit cell contains six formulas with a space group of $\mathrm{R} \overline{3} \mathrm{c}$. Computational studies of first principle calculation have used $\mathrm{Li}_{1.17} \mathrm{Al}_{0.17} \mathrm{Ti}_{1.83}\left(\mathrm{PO}_{4}\right)_{3}$ (seven $\mathrm{Li}$ atoms) [11] and $\mathrm{LiTi}_{2}\left(\mathrm{PO}_{4}\right)_{3}$ (six Li atoms) [12] to represent LATP. In this study, we chose $\mathrm{Li}_{1.17} \mathrm{Al}_{0.17} \mathrm{Ti}_{1.83}\left(\mathrm{PO}_{4}\right)_{3}$ as its ratio is close to the one that has the highest ionic conductivity and also simplifies the distribution of Li atoms. It has been studied that there are two sites for Li atoms, noted as Li(1) and $\operatorname{Li}(2)$. $\operatorname{Li}(1)$ has been found to be fully occupied at $6 \mathrm{~b}$ sites, but the $\mathrm{Li}(2)$ sites have been argued and claimed to be at $6 \mathrm{a}[9]$ or $36 \mathrm{f}[10,13]$ site. The $36 \mathrm{f}$ site for $\mathrm{Li}$ atom was picked in this study as both experimental and computational results confirmed it. One of the Ti atoms in $\mathrm{LiTi}_{2}\left(\mathrm{PO}_{4}\right)_{3}$ was randomly chosen to be replaced by $\mathrm{Al}$ atom, and the $\mathrm{Li}$ was placed at the 36 sites around it, which was based on the computational result [13]. 
To the best of our knowledge, no computational or experimental study has revealed the most stable LATP surface structure. Same as the preserved $-\mathrm{PS}_{4}$ polyhedral in $\beta-\mathrm{Li}_{3} \mathrm{PS}_{4}$ surface structure, keeping the -PO4 polyhedral was the principle when cleaving the surfaces. Under the condition, (001) and (012) directions were chosen, and the two $\mathrm{Al}$ atoms were distributed in two different situations, where they both stay at the surface or bulk to maintain a symmetrical slab for calculating the surface energies, as Figure S2 shows the testing atomic surface structures. The selected (012) direction is same as the lattice plane that was shown in a high-resolution TEM (HRTEM) image of one nanocube edge of NASICON-NaTi $2\left(\mathrm{PO}_{4}\right)_{3}[14]$, which consisted an interplanar distance of $6.1 \AA$ that is also similar to the distance of Ti-Ti $(6.0 \AA)$ along (012) direction of LATP. Several different symmetrical terminations of the slabs, such as $\mathrm{O}, \mathrm{Li}, \mathrm{Ti}$, and $\mathrm{Al}$ have been calculated along these two orientations for the surface energies to evaluate the most stable surface structure. Among those, only the (012) direction is possible to have a stoichiometric and symmetrical slab that is terminated by $\mathrm{Li}$ atoms after deleting the surface $\mathrm{O}$ atoms (from $\mathrm{TiO}_{6}$ blocks) and $50 \%$ of the Li atoms with $\mathrm{Al}$ distributed on the surface layer, and it also has a relatively small surface energy of $0.37 \mathrm{~J} / \mathrm{m}^{2}$. Therefore, it was selected to represent the LATP surface structure.

\section{LiPON}

The ALD-LiPON composition has been determined as $\mathrm{Li}_{2} \mathrm{PO}_{2} \mathrm{~N}$ [15], also called SD-Li $\mathrm{PO}_{2} \mathrm{~N}$. Its atomic structure has been discussed [16] and was taken for this study as well. A unit cell of bulk $\mathrm{Li}_{2} \mathrm{PO}_{2} \mathrm{~N}$ consists of 4 formulas with a space group of $\mathrm{Cmc}_{1}$. The stoichiometric surface structure was taken from the literature $[5,17]$, which was cut along the direction (100) of bulk structure and preserved the $\mathrm{PN}_{2} \mathrm{O}_{2}$ polyhedral and terminated symmetrically with $\mathrm{Li}$ atoms on both ends. Four $\left(\mathrm{Li}_{16} \mathrm{P}_{8} \mathrm{O}_{16} \mathrm{~N}_{8}\right)$ or more layers of -P-N-P- is enough for the convergence of the surface energy. 
The final and the most energetic-favorable surface structures used in later DOS and charge density calculation are shown in Fig. S1.

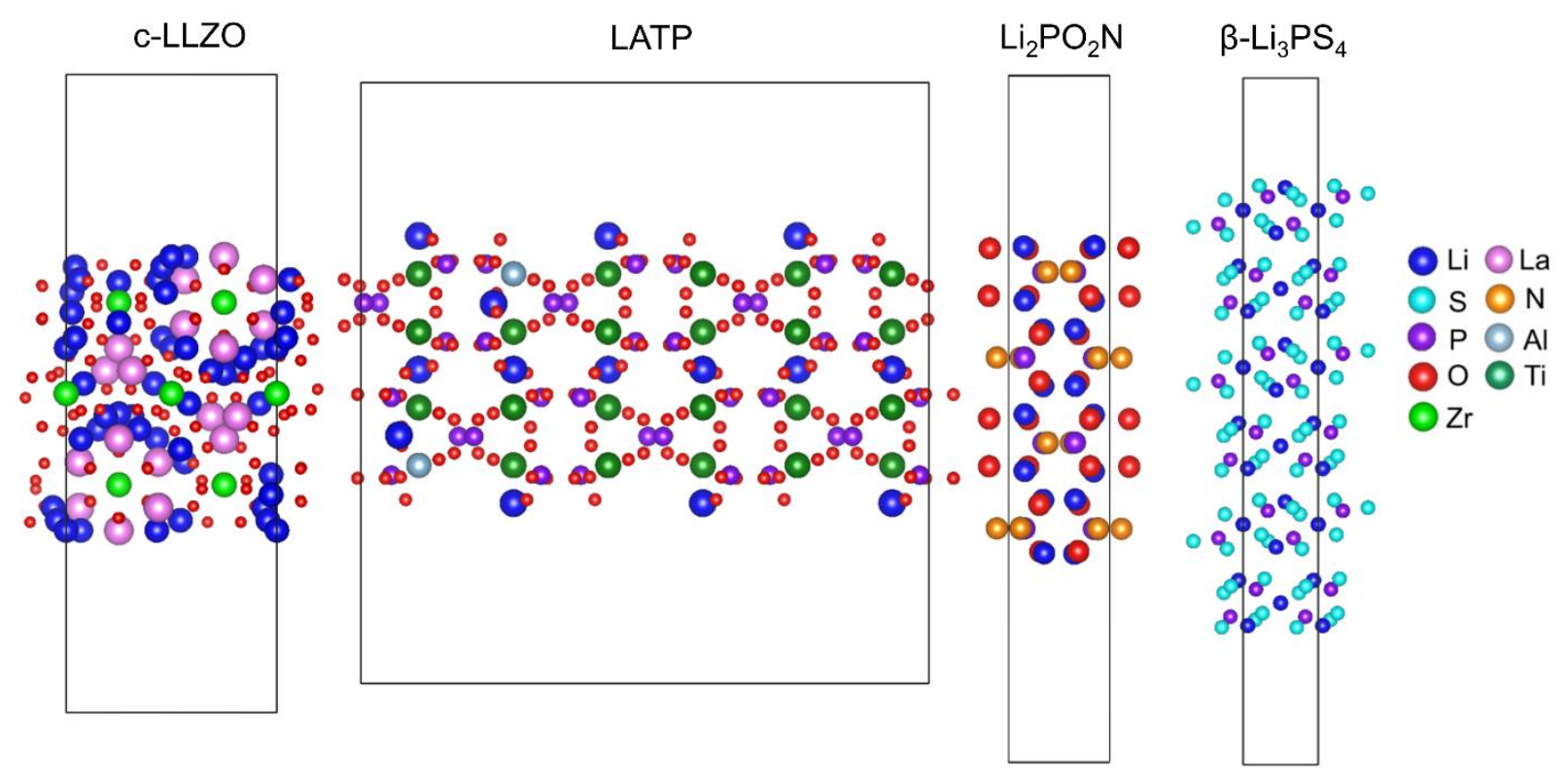

Figure S1. The most energetic-favorable surface structure for different solid electrolytes. 


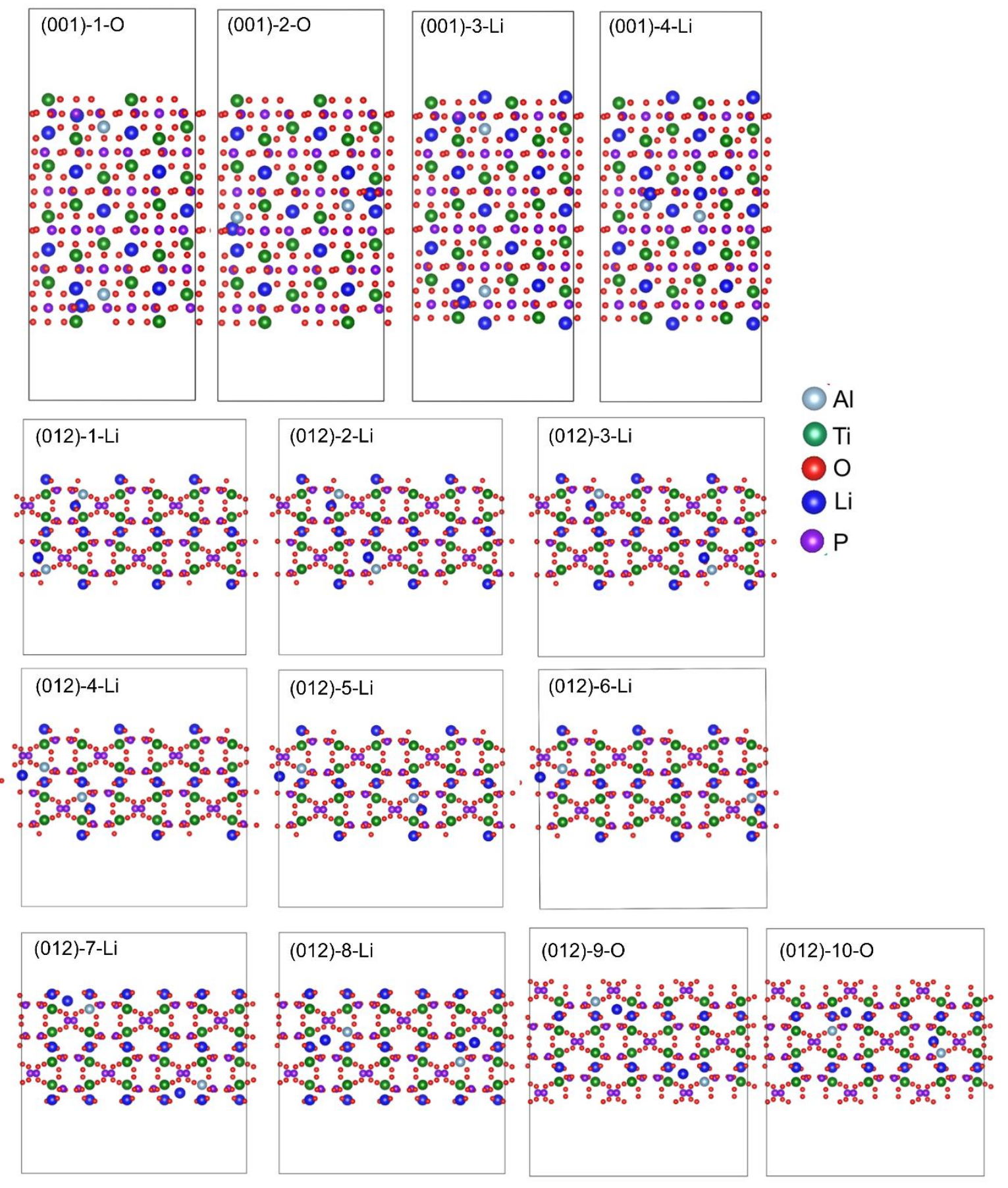

Figure S2. LATP surface structures with different orientations, terminations, and Al distributions, detailed calculation results are listed in Table S1 and Table S2. 


\section{I.2 Surface energy}

First-principles calculations were performed via the Vienna Ab initio Simulation Package (VASP) [18] based on plane-wave DFT. The surface calculations were mainly performed with the semilocal generalized gradient approximation (GGA) [19] of Perdew, Burke, and Emzerhof (PBE) [20]. Since the $\mathrm{Zr}$ in c-LLZO and the Ti in LATP are early-transition metals, it may not be necessary to apply Hubbard-type correction $U$, as of how the previous studies calculated for these two materials $[21,22]$. The surface energy calculation was the same ass the previous studies $[5,21]$ that included the correction of chemical potential for the excess/deficient atoms, as Equation S1 shows.

$$
\gamma=\frac{1}{2 A}\left(E_{\text {slab }}-n_{\text {formula }} * \mu_{\text {bulk }}-\sum n_{i} \mu_{i}\right)
$$

where $A$ is the cross-section area, $E_{\text {slab }}$ is the total energy of the slab, $n_{\text {formula }}$ is the integer number of stoichiometric formula units in the slab, $\mu_{b u l k}$ is the energy of one formula unit of corresponding bulk structure, $n_{i}$ is the number of atoms of type $i$ in the slab in excess of the stoichiometric amount. $\mu_{i}$ is the chemical potential of element $i$, which was tabulated from the total energy of its equilibrium phases against Li metal. For example, the equilibrium phases of LATP vs. $\mathrm{Li}$ are $\mathrm{Ti}_{3} \mathrm{P}, \mathrm{TiAl}, \mathrm{Li}_{3} \mathrm{P}$ and $\mathrm{Li}_{2} \mathrm{O}$ [11] and the relationship between the total energy/formula, $E$, and their chemical potential, $\mu_{i}$ could be represented as:

$$
\begin{aligned}
& E\left(\mathrm{Ti}_{3} \mathrm{P}\right)=3 \mu_{\mathrm{Ti}}+\mu_{\mathrm{P}} \\
& E(\mathrm{TiAl})=\mu_{\mathrm{Ti}}+\mu_{\mathrm{Al}} \\
& E\left(\mathrm{Li}_{3} \mathrm{P}\right)=3 \mu_{\mathrm{Li}}+\mu_{\mathrm{P}} \\
& E\left(\mathrm{Li}_{2} \mathrm{O}\right)=2 \mu_{\mathrm{Li}}+\mu_{\mathrm{O}} \\
& E\left(\mathrm{Li}_{\mathrm{BCC}} \text { metal }\right)=\mu_{\mathrm{Li}}
\end{aligned}
$$


The chemical potential of each element of c-LLZO was obtained from the literature [21] and listed with our tabulated results of LATP in Table S1. The calculated results of surface energy for different LATP surface structures are listed in Table S2, and we chose 012-1-Li as the final surface structure of LATP.

Table S1. Chemical potentials (eV/atom) used in surface energy calculations.

\begin{tabular}{cccccc}
\hline & $\mathrm{Li}$ & $\mathrm{La}$ & $\mathrm{Zr}$ & $\mathrm{O}$ & \\
c-LLZO[21] & -1.904 & -5.165 & -8.481 & -10.542 & \\
\hline & $\mathrm{Li}$ & $\mathrm{Al}$ & $\mathrm{Ti}$ & $\mathrm{P}$ & $\mathrm{O}$ \\
LATP & -1.904 & -2.537 & -8.111 & -7.931 & -10.377 \\
\hline
\end{tabular}

Table S2. Comparison of the surface energy for different surface structures of LATP

\begin{tabular}{|c|c|c|c|c|}
\hline Name & $\begin{array}{l}\text { Stoichiometric or } \\
\text { nonstoichiometric? }\end{array}$ & Atomic ratio & $\mathrm{Al}$ position & $\begin{array}{l}\text { Surface energy } \\
\quad\left(\mathrm{J} \mathrm{m}^{-2}\right)\end{array}$ \\
\hline $001-1-O$ & Nonstoichiometric & $\mathrm{Li}_{12} \mathrm{Al}_{2} \mathrm{Ti}_{22} \mathrm{P}_{36} \mathrm{O}_{144}$ & top-bottom & 1.4394 \\
\hline $001-2-\mathrm{O}$ & Nonstoichiometric & $\mathrm{Li}_{12} \mathrm{Al}_{2} \mathrm{Ti}_{22} \mathrm{P}_{36} \mathrm{O}_{144}$ & middle & 1.2068 \\
\hline 001-3-Li & Nonstoichiometric & $\mathrm{Li}_{16} \mathrm{Al}_{2} \mathrm{Ti}_{22} \mathrm{P}_{36} \mathrm{O}_{144}$ & top-bottom & 0.4282 \\
\hline 001-4-Li & Nonstoichiometric & $\mathrm{Li}_{16} \mathrm{Al}_{2} \mathrm{Ti}_{22} \mathrm{P}_{36} \mathrm{O}_{144}$ & middle & 0.4258 \\
\hline 012-1-Li & Stoichiometric & $\mathbf{L i}_{14} \mathrm{Al}_{2} \mathrm{Ti}_{22} \mathbf{P}_{36} \mathbf{O}_{144}$ & top-bottom-1 & 0.3743 \\
\hline 012-2-Li & Stoichiometric & $\mathbf{L i}_{14} \mathrm{Al}_{2} \mathrm{Ti}_{22} \mathbf{P}_{36} \mathbf{O}_{144}$ & top-bottom-2 & 0.3741 \\
\hline 012-3-Li & Stoichiometric & $\mathbf{L i}_{14} \mathrm{Al}_{2} \mathrm{Ti}_{22} \mathbf{P}_{36} \mathrm{O}_{144}$ & top-bottom-3 & 0.3736 \\
\hline 012-4-Li & Stoichiometric & $\mathrm{Li}_{14} \mathrm{Al}_{2} \mathrm{Ti}_{22} \mathrm{P}_{36} \mathrm{O}_{144}$ & middle-1 & 0.4722 \\
\hline $012-5-\mathrm{Li}$ & Stoichiometric & $\mathrm{Li}_{14} \mathrm{Al}_{2} \mathrm{Ti}_{22} \mathrm{P}_{36} \mathrm{O}_{144}$ & middle-2 & 0.4732 \\
\hline 012-6-Li & Stoichiometric & $\mathrm{Li}_{14} \mathrm{Al}_{2} \mathrm{Ti}_{22} \mathrm{P}_{36} \mathrm{O}_{144}$ & middle-3 & 0.4727 \\
\hline 012-7-Li & Nonstoichiometric & $\mathrm{Li}_{20} \mathrm{Al}_{2} \mathrm{Ti}_{22} \mathrm{P}_{36} \mathrm{O}_{156}$ & top-bottom & 2.6460 \\
\hline 012-8-Li & Nonstoichiometric & $\mathrm{Li}_{20} \mathrm{Al}_{2} \mathrm{Ti}_{22} \mathrm{P}_{36} \mathrm{O}_{156}$ & middle & 2.7123 \\
\hline 012-9-O & Nonstoichiometric & $\mathrm{Li}_{14} \mathrm{Al}_{2} \mathrm{Ti}_{22} \mathrm{P}_{42} \mathrm{O}_{180}$ & top-bottom & 2.3751 \\
\hline $012-10-\mathrm{O}$ & Nonstoichiometric & $\mathrm{Li}_{14} \mathrm{Al}_{2} \mathrm{Ti}_{22} \mathrm{P}_{42} \mathrm{O}_{180}$ & middle & 2.1878 \\
\hline
\end{tabular}




\section{I.3 Electronic Structure Analysis}

Core-valence electron interaction was treated using projector augmented wave (PAW) [23], and two different levels of theory were implemented: the semi-local GGA [19] of PBE [20] and the hybrid functional of Heyd, Scuseria, and Ernzerhof (HSE06) [24, 25]. The HSE06 calculation results about the bandgap information are listed in Table 1, and the TDOS is plotted in Figure S3. Since the Zr in c-LLZO and the Ti in LATP are early-transition metals, it may not be necessary to apply Hubbard-type correction U, as of how the previous studies calculated for these two materials $[21,22]$. 

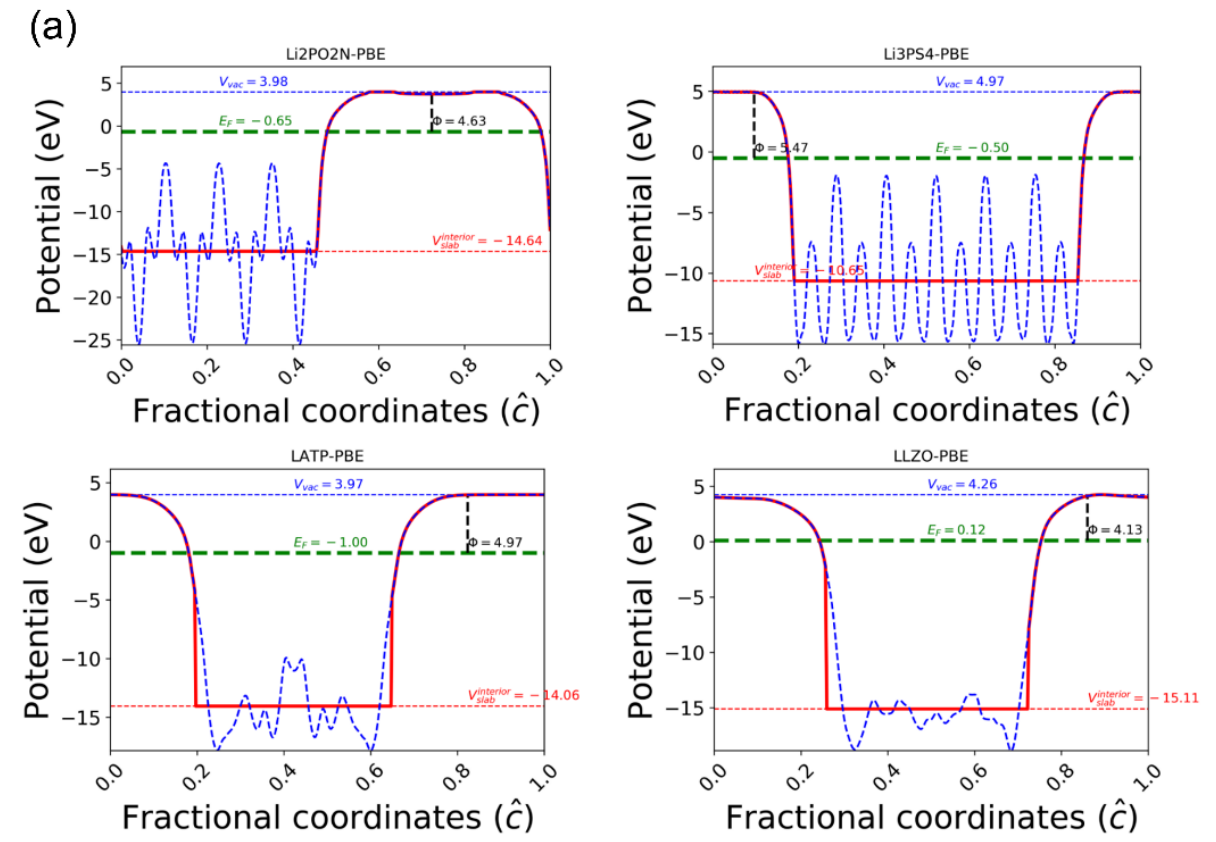

(b)
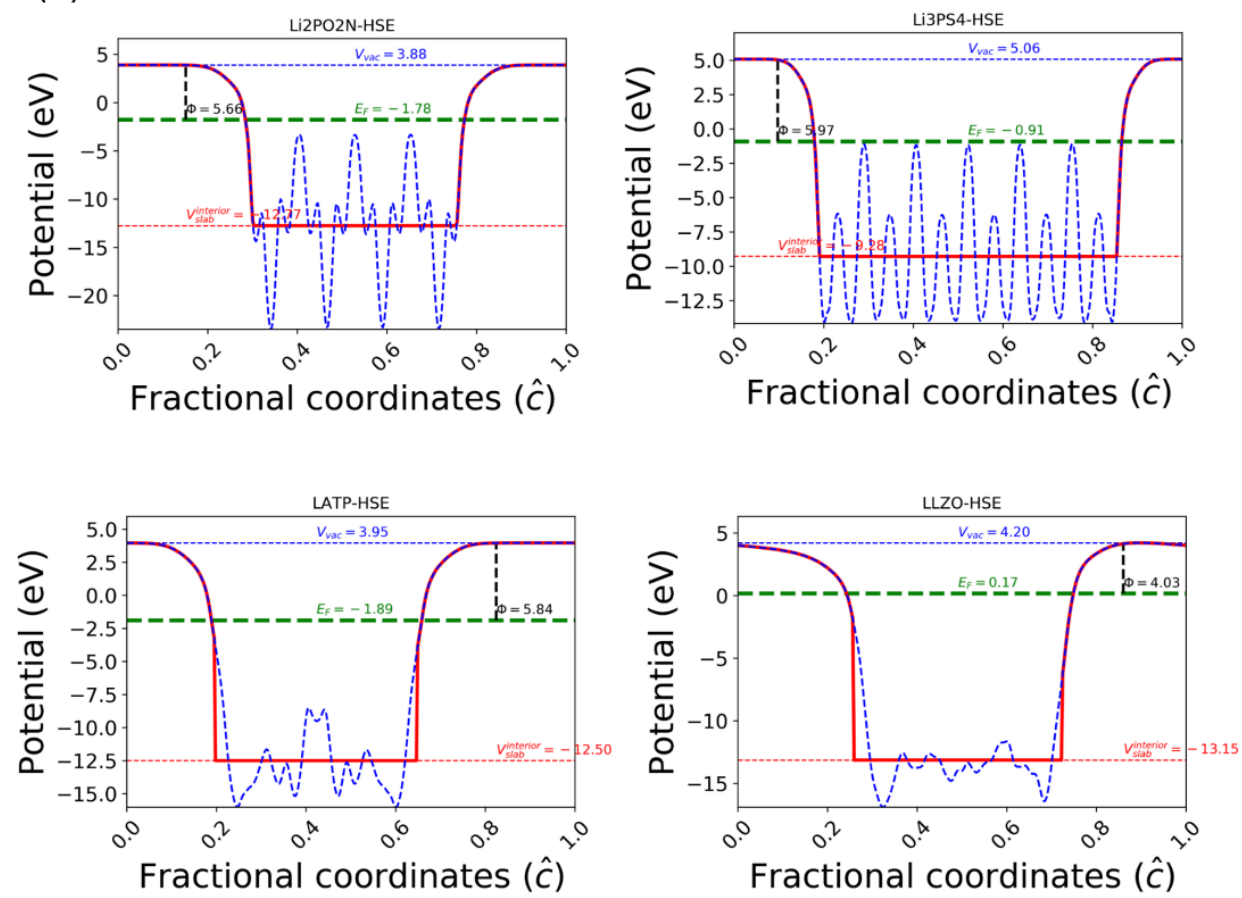

Figure S3. Electron potential in slab cell for different materials from (a) GGA-PBE and (b) HSE06 calculations. $\mathrm{V}_{\mathrm{vac}}$ stands for the potential at vacuum, $\mathrm{E}_{\mathrm{F}}$ is the Fermi level, $\mathrm{V}_{\text {slab }}^{\text {interior }}$ is the potential in slab, and $\Phi$ is the work function. 


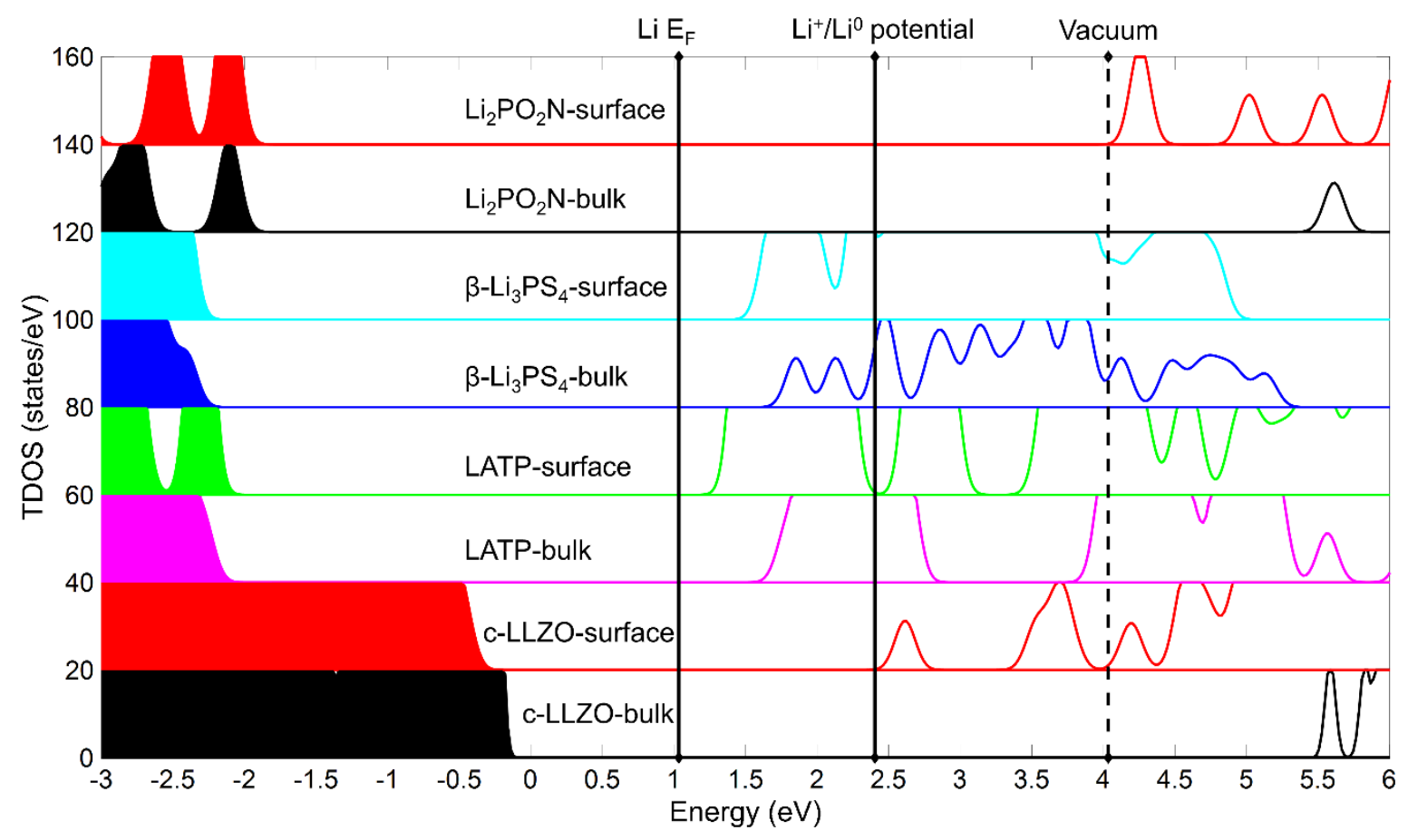

Figure S4. Total density of states calculated by DFT HSE06 calculation. Li EF stands for the Fermi level of Li metal, the vacuum level is plotted as vertical dashed line.

The charge density difference was evaluated before and after the additional electrons were added to the relaxed surface structures (slab with extra electrons minus neutral slab. The amount of inserted electrons was normalized by the cross-section area (along c-axis) of the slab and listed in Table S3.

Table S3. Amount of additional electrons in each slab based on difference cross-section area.

\begin{tabular}{cccc}
\hline Surface structures & $\begin{array}{c}\text { Cross-section area } \\
\text { per unit cell }\left(\AA^{2}\right)\end{array}$ & Excess electrons & $\begin{array}{c}\text { Excess electrons per unit area } \\
\text { at the surface }\left(\# / \AA^{2}\right)\end{array}$ \\
\hline c-LLZO & 118.23 & 0.5460 & \\
LATP & 216.54 & 1 & 0.0046 \\
$\beta-\mathrm{Li}_{3} \mathrm{PS}$ & 50.31 & 0.2323 & \\
$\mathrm{Li}_{2} \mathrm{PO}_{2} \mathrm{~N}$ & 25.30 & 0.1168 & \\
\hline
\end{tabular}




\section{Phase Field Modeling}

\section{II.1 Formulating the total energy}

The total energy $\mathcal{F}$ of the inhomogeneous system is given by

$\mathcal{F}=\int\left[f_{\text {local }}\left(\xi, \phi_{g}, \tilde{c}_{i}\right)+f_{\text {grad }}\left(\nabla \xi, \nabla \phi_{g}\right)+f_{\text {elec }}\left(c_{i}, \varphi\right)+f_{\text {mech }}\left(\xi, \phi_{g}\right)\right] d V$

where $\tilde{c}_{i}=c_{i} / c_{0}$ is a set of dimensionless concentrations of species, (i.e. $i=L i^{+}, e^{-}$) where $c_{0}$ is the bulk concentration of the solid phase. $\varphi$ is the electrostatic potential. The local energy density, $f_{\text {local }}$, is further written as $f_{\text {local }}=f_{0}\left(\xi, \phi_{g}\right)+c_{0} R T \sum_{i} \tilde{c}_{i} \ln \tilde{c}_{i}+\sum_{i} c_{i} \mu_{i}^{\Theta}$, where $R, T$ and $\mu_{i}^{\Theta}$ are gas constant, temperature and the standard chemical potential of species $i$, respectively. $f_{0}$ is formulated as

$f_{0}=A \xi^{2}(1-\xi)^{2}+\sum_{g=1}^{N} B \phi_{g}{ }^{2}\left(1-\phi_{g}\right)^{2}+\sum_{p=1}^{N} \sum_{q \neq p}^{N} \frac{C}{2}{\phi_{p}}^{2}{\phi_{q}}^{2}+\sum_{g=1}^{N} \frac{D}{2} \xi^{2} \phi_{g}{ }^{2}$,

where the phenomenological parameters are set to be $A=B=1$ and $C=D=3$, so that the equilibrium states are represented by the degenerate minima with equal depth on the landscape of $f_{0}$ at values of 0 or 1 for the phase-field variables. The gradient energy density is in the form of $f_{\text {grad }}=\frac{1}{2} \kappa_{\xi}(\nabla \xi)^{2}+\sum_{g=1}^{N} \frac{1}{2} \kappa_{\phi}\left(\nabla \phi_{g}\right)^{2}-\kappa_{G B} \sum_{p=1}^{N} \sum_{q \neq i}^{N} \nabla \phi_{p} \nabla \phi_{q}$, where $\kappa_{\xi}, \kappa_{\phi}$ and $\kappa_{G B}$ are the gradient coefficients associated with Li metal surface energy, solid electrolyte surface energy, and solid electrolyte GB adhesion energy, respectively. The surface energy anisotropy of Li metal is incorporated by $\kappa_{\xi}=\kappa_{0}[1+\delta \cos (\omega \theta)]^{2}$, where $\kappa_{0}$ is related to the average Li metal surface energy and interface width, $\delta, \omega$ and $\theta$ are the anisotropy strength, crystallographic symmetry mode ( $\omega=4$ for body-centered cubic Li metal), and the angle between the reference axis and the normal direction of surface migration, respectively. The current model assumes isotropic solid electrolyte properties, and thus constant values are used for $\kappa_{\phi}$ and $\kappa_{G B} . f_{\text {elec }}=F \sum_{i} z_{i} c_{i} \varphi$ is the 
electrostatic energy density, where $z_{i}$ is the valence of species and $F$ is the Faraday constant. The elastic energy density is expressed as $f_{m e c h}=\frac{1}{2} C_{i j k l}\left(\xi, \phi_{g}\right)\left(\varepsilon_{i j}-\varepsilon_{i j}^{0}\right) \cdot\left(\varepsilon_{k l}-\varepsilon_{k l}^{0}\right)$, where the effective stiffness of the system is given by $C_{i j k l}\left(\xi, \phi_{g}\right)=\left[h(\xi) C_{i j k l}^{\xi}+\sum_{n=1}^{N} h\left(\phi_{n}\right) C_{i j k l}^{\phi}\right]$, interpolated by function $h(\xi)=\xi^{3}\left(10-15 \xi+6 \xi^{2}\right) \cdot C_{i j k l}^{\xi}$ and $C_{i j k l}^{\phi}$ are the corresponding elastic stiffness tensors of $\mathrm{Li}$ metal and solid electrolyte, respectively. Under the isotropic assumption, the eigen strain is written as $\varepsilon_{x x}^{0}=\varepsilon_{y y}^{0}=\varepsilon_{z z}^{0}=\sum_{g=1}^{N} \xi \phi_{g}^{0}\left(\Delta V_{\phi_{g}}\right)^{-3}$, where $\phi_{g}^{0}$ and $\Delta V_{\phi_{g}}$ are the initial field value and the volumetric change compared to the initial volume of the $g$ th grain, respectively.

\section{II.2 Mass transport and electrostatic distribution}

The transport of Li ion in the system is governed by Nernst-Planck equation,

$\frac{\partial \tilde{c}_{L i+}}{\partial t}=\nabla \cdot\left[D \nabla \tilde{c}_{L i+}+\frac{D \tilde{L}_{L i+}}{R T} F \nabla \varphi\right]-\frac{c_{S}}{c_{0}} \frac{\partial \xi}{\partial t}$

where the effective Li ion diffusion coefficient $D$ of the system is interpolated as $D=$ $D_{\text {anode }} h(\xi)+\sum_{g=1}^{N} D_{S E} h\left(\phi_{g}\right) . D_{\text {anode }}$ and $D_{S E}$ are the Li ion diffusion coefficients in Li metal anode and solid electrolyte bulk, respectively (assume $D_{\text {anode }} \ll D_{S E}$ ). The last term is the electrodeposition reaction induced $\mathrm{Li}$ ion consumption/production at the electrode/electrolyte interface, where $c_{s}=7.64 \times 10^{4} \mathrm{~mol} \cdot \mathrm{m}^{-3}$ is the site density of Li metal. The electrostatic potential distribution is described by a Poisson-type equation with a reaction rate-related term accounting for the temporal charge annihilation/generation at anode surface:

$\nabla \cdot[\sigma \cdot \nabla(\varphi(\boldsymbol{r}, t))]=I_{R}$

where the effective conductivity of the system, $\sigma$, is interpolated as $\sigma=\sigma_{\text {anode }} h(\xi)+$ $\sum_{g=1}^{N} \sigma_{S E} h\left(\phi_{g}\right)$, in which $\sigma_{\text {anode }}$ and $\sigma_{S E}$ are the conductivities of Li metal anode and solid 
electrolyte bulk, respectively. The source term, $I_{R}=n F c_{s} \frac{\partial \xi}{\partial t}$, is correlated with the electrodeposition reaction rate. A detailed description of the Li ion transport and electrostatic part of the model can be found in reference [26].

\section{II.3 Incorporating SE surface excess electron impact}

According to the previous study [5], the surface LLZO could be either stoichiometric or nonstoichiometric since both have similar surface energies. Not only the lower bandgap $(2.3 \mathrm{eV})$ from the stoichiometric surface would get more electrons in conduction bands than bulk, but also the non-stoichiometric surface (Li segregated) would have metal-like behavior because the Fermi level has been lifted to conduction bands. This implies there are some electrons would transfer from anode to LLZO surfaces. Even though, the surfaces could still maintain neutral since the measured electrical conductivity $\left(\sim 10^{-8} \mathrm{~S} \mathrm{~cm}^{-1}\right)$ [27] is much lower than the measured Li-ion conductivity $\left(\sim 10^{-4} \mathrm{~S} \mathrm{~cm}^{-1}\right)$ [28], it is expected that the Li-ion could sufficiently compensate the charges of additional electrons on the LLZO surfaces. This amplifies the effect of trapped electrons on the extended internal defects, or a "worst" scenario for Li-dendrite nucleation. Therefore, to estimate the intrinsic surface electron concentration in LLZO, we could use the density of states of nonstoichiometric LLZO surface as input for the phase-field model and assume constant equilibrium electronic surface states during electrodeposition. The electron distribution of a given system is described as:

$n=\int_{-\infty}^{+\infty} F(\varepsilon) D(\varepsilon) d \varepsilon=\int_{-\infty}^{+\infty} \frac{D(\varepsilon)}{1+e^{\left(\varepsilon-\varepsilon_{F}\right) / k_{b} T}} d \varepsilon$ 
where $\varepsilon$ is the energy of states, $F(\varepsilon)$ is the Fermi-Dirac distribution, $D(\varepsilon)$ is the density of states per volume in the unit of $\left[\left(e V \cdot \AA^{3}\right)^{-1}\right], \varepsilon_{F}$ is the Fermi level, $k_{b}$ is the Boltzmann constant and $T$ is the temperature. The surface trapped electron concentration at room temperature, $c_{s u r f}$, can thus be estimated with the $0 \mathrm{~K}$ DFT data and a temperature correction factor $\left(\lambda_{T}\right)$, by integrating at the Fermi level,

$c_{\text {surf }} \approx n_{F e r m i}=\int_{\varepsilon_{F}-k_{b} T}^{\varepsilon_{F}+k_{b} T} \frac{\lambda_{T} D\left(\varepsilon_{F}\right)}{1+e^{\left(\varepsilon_{F}-\varepsilon_{F}\right) / k_{b} T}} d \varepsilon \approx 2 k_{b} T \frac{\lambda_{T} D\left(\varepsilon_{F}\right)}{2}=\lambda_{T} D\left(\varepsilon_{F}\right) k_{b} T$

Taking unit value for $\lambda_{T}$ and DFT data of $D\left(\varepsilon_{F}\right)$, the concentration of the trapped electrons on the surface of nonstoichiometric c-LLZO was calculated to be around $0.337 \mathrm{~mol} / \mathrm{L}$. [5]. Since the focus is on the intrinsic and static material properties on the internal defects in SE, we did not consider how the Fermi level changes with the applied voltage.

The electron concentration decays sharply from solid electrolyte surface into bulk, which can be described using the calculated concentrations of surface trapped electrons, $c_{s u r f}$, and the phasefield order parameters in the form of

$c_{e}(x, t)=\xi+\sum_{i} c_{\text {surf }}(1-\xi)^{2}\left(1-\phi_{i}\right) \chi_{i}$

where $\xi$ and $\phi_{i}$ are the phase-field order parameters identifying the Li metal phase and $i$ th solid electrolyte grain, respectively. $\chi_{i}$ is a step function confining the electrons within the $i$-th solid electrolyte grain surface region,

$\chi_{i}=\left\{\begin{array}{l}1,\left|\phi_{i}-0.5\right|<h \\ 0, \text { otherwise }\end{array}\right.$

where $h$ is a surface thickness parameter that equals 0.05 in this work. 


\section{II.4 Li dendrite nucleation rate}

Based on classical nucleation theory, the nucleation rate of the precipitated phase can be generally written as,

$J^{*}=J_{0}^{*} \exp \left(-\frac{\sigma_{\text {inhomo }} \Delta G^{*}}{k_{b} T}\right)$

where $J_{0}^{*}$ is an empirical kinetic pre-factor. Given the complexity and randomness of the polycrystalline solid electrolyte structure, we introduced a correction factor, $\sigma_{\text {inhomo }}$, to describe the local inhomogeneity deviated from classical nucleation, such that both homogeneous and heterogeneous scenarios were considered in the model. $\Delta G^{*}$ is the nucleation activation energy to form a stable nucleus. The total energy variation during a classical nucleation process is written as

$\Delta G=-\left(\frac{\Delta \mu}{V_{m}}\right) \cdot \frac{4}{3} \pi r^{3}+4 \pi r^{2} \cdot \gamma$

where $\Delta \mu$ and $\gamma$ are the chemical potential difference and interfacial energy between matrix and precipitate phase, respectively. $V_{m}$ is the molar volume of the precipitate phase and $r$ is the nuclei radius. $\Delta G^{*}$ is determined by the saddle point of $\Delta G$ at critical radius $r^{*}=\frac{2 \gamma V_{m}}{\Delta \mu}$, where the interfacial energy increase just gets compensated by the free energy decrease of phase transition,

$\Delta G^{*}=\left.\Delta G\right|_{r=r^{*}}=\frac{16 \pi \gamma^{3} V_{m}^{2}}{3 \Delta \mu^{2}}$

During Li electrodeposition process, the electrochemical potential difference between precipitated Li nuclei and solid electrolyte matrix is given by $\Delta \bar{\mu}=-R T \ln \frac{\tilde{c}_{L i+} \tilde{c}_{e}}{a_{L i}}-F\left(E^{\Theta}-\Delta \varphi\right)=F \eta$, where $a_{L i}$ is the activity of Li metal atom (treated as 1 in this work), $E^{\Theta}$ is the standard half-cell potential, and $\Delta \varphi$ is the interfacial potential difference between precipitated Li metal and solid electrolyte phase. Therefore, the nucleation rate can be rewritten as 
$J^{*}=J_{0}^{*} \exp \left(-\frac{16 \sigma_{\text {inhomo } o} \pi \gamma^{3} V_{m}^{2}}{3 F^{2} \eta^{2} k_{b} T}\right)$

The detailed derivation of Li electrodeposition thermodynamics can be found in reference [26].

\section{II.5 Phase-field simulation parameter table}

Table S4. Phase-field simulation parameters and their normalizations.

\begin{tabular}{|c|c|c|c|c|c|c|c|}
\hline & \multirow{2}{*}{ Symbol } & \multirow{2}{*}{ Unit } & \multicolumn{2}{|c|}{ Real Value } & \multirow{2}{*}{ Normalization } & \multicolumn{2}{|c|}{ Normalized Value } \\
\hline & & & $\mathrm{Li}$ & LLZO & & $\mathrm{Li}$ & LLZO \\
\hline System Size & $l$ & $m$ & \multicolumn{2}{|c|}{$5.0 \times 10^{-4}$} & $\tilde{l}=l / l_{0}$ & \multicolumn{2}{|c|}{5} \\
\hline Time step & $\Delta t$ & $s$ & \multicolumn{2}{|c|}{0.2} & $\Delta \tilde{t}=\Delta t / t_{0}$ & \multicolumn{2}{|c|}{$5.0 \times 10^{-5}$} \\
\hline Interface mobility & $L_{\sigma}$ & $m^{3}(J \cdot s)^{-1}$ & $2.5 \times 10^{-6}$ & $1.5 \times 10^{-8}$ & $\tilde{L}_{\sigma}=L_{\sigma} \cdot\left(E_{0} \cdot t_{0}\right)$ & $1.5 \times 10^{5}$ & $9.0 \times 10^{2}$ \\
\hline Barrier Height & $W$ & $J \cdot m^{-3}$ & $1.18 \times 10^{6}$ & $2.05 \times 10^{6}$ & $\widetilde{W}=W / E_{0}$ & 0.08 & 0.14 \\
\hline Gradient Coefficient & $\kappa_{0}$ & $J \cdot m^{-1}$ & $3.68 \times 10^{-6}$ & $6.38 \times 10^{-6}$ & $\tilde{\kappa}_{0}=\kappa_{0} \cdot\left(E_{0} / l_{0}^{2}\right)$ & $2.45 \times 10^{-5}$ & $4.25 \times 10^{-5}$ \\
\hline Anisotropy & $\delta$ & - & 0.03 & 0 & - & 0.03 & 0 \\
\hline $\mathrm{Li}^{+}$Diffusion Coefficient & $D$ & $m^{2} \cdot s^{-1}$ & $1.0 \times 10^{-15}$ & $1.0 \times 10^{-12}$ & $\widetilde{D}=D /\left(l_{0}^{2} / \Delta t_{0}\right)$ & $4.0 \times 10^{-4}$ & 0.4 \\
\hline Conductivity & $\sigma$ & $S \cdot m^{-1}$ & $1.0 \times 10^{7}$ & $1.0 \times 10^{-5}$ & $\tilde{\sigma}=\sigma \cdot\left(\Delta t_{0} R T\right) /\left(c_{0} F^{2} l_{0}^{2}\right)$ & $2.51 \times 10^{7}$ & $2.51 \times 10^{-5}$ \\
\hline Young's Modulus & $E$ & $J \cdot m^{-3}$ & $4.9 \times 10^{9}$ & $1.5 \times 10^{11}$ & $\tilde{E}=E / E_{0}$ & $3.27 \times 10^{2}$ & $1.00 \times 10^{4}$ \\
\hline Poisson Ratio & $v$ & - & 0.362 & 0.257 & - & 0.362 & 0.257 \\
\hline Electrochemical pre-factor & $L_{\eta}$ & $s^{-1}$ & 0.005 & - & $\tilde{L}_{\eta}=L_{\eta} \cdot t_{0}$ & 20 & - \\
\hline Symmetric factor & $\alpha$ & - & 0.5 & - & - & 0.5 & - \\
\hline Nucleation rate pre-factor & $J_{0}^{*}$ & - & 200 & - & - & 200 & - \\
\hline Inhomo correction factor & $\sigma_{\text {inhomo }}$ & - & 1 & - & - & 1 & - \\
\hline
\end{tabular}

Phase-field simulation input parameters were normalized by a characteristic length $l_{0}=1 \times$ $10^{-4} \mathrm{~m}$, a characteristic energy density $E_{0}=1.5 \times 10^{7} \mathrm{~J} \cdot \mathrm{m}^{-3}$, the Li ion bulk concentration of 
LLZO $c_{0}=42.2 \times 10^{3} \mathrm{~mol} \cdot \mathrm{m}^{-3}$, and a characteristic time step $\Delta t_{0}=4 \times 10^{3} \mathrm{~s}$ at room temperature $T=298 \mathrm{~K}$.

\section{II.6 Additional phase-field simulation results}

The impacts of surface trapped electrons are dynamically demonstrated by the comparison of the morphology, $\mathrm{Li}$ ion concentration and electric potential evolution snapshots during the $\mathrm{Li}$ electrodeposition processes in the $75 \mu m$ grain size "ideal" LLZO (Figure S3) and real LLZO (Figure S4) half cells, respectively. From the morphology evolution figures, the Li intergranular growth is dramatically accelerated in the real LLZO system (Figure S4 a-e) than the ideal LLZO system (Figure S3 a-e), which is due to the increased electrochemical driving force contributed from the trapped surface electrons as well as the additional dendrite growth sites at the isolated nucleation spots within the healthy SE region, as mentioned in the main text. Meanwhile, the isolatedly formed Li metal will hinder or even shut down the intergranular Li ion transport (Figure S4 j-g) and lead to the sudden decrease of local electric potential (Figure S4 1-o), which impedes the normal electrodeposition at anode substrate. 

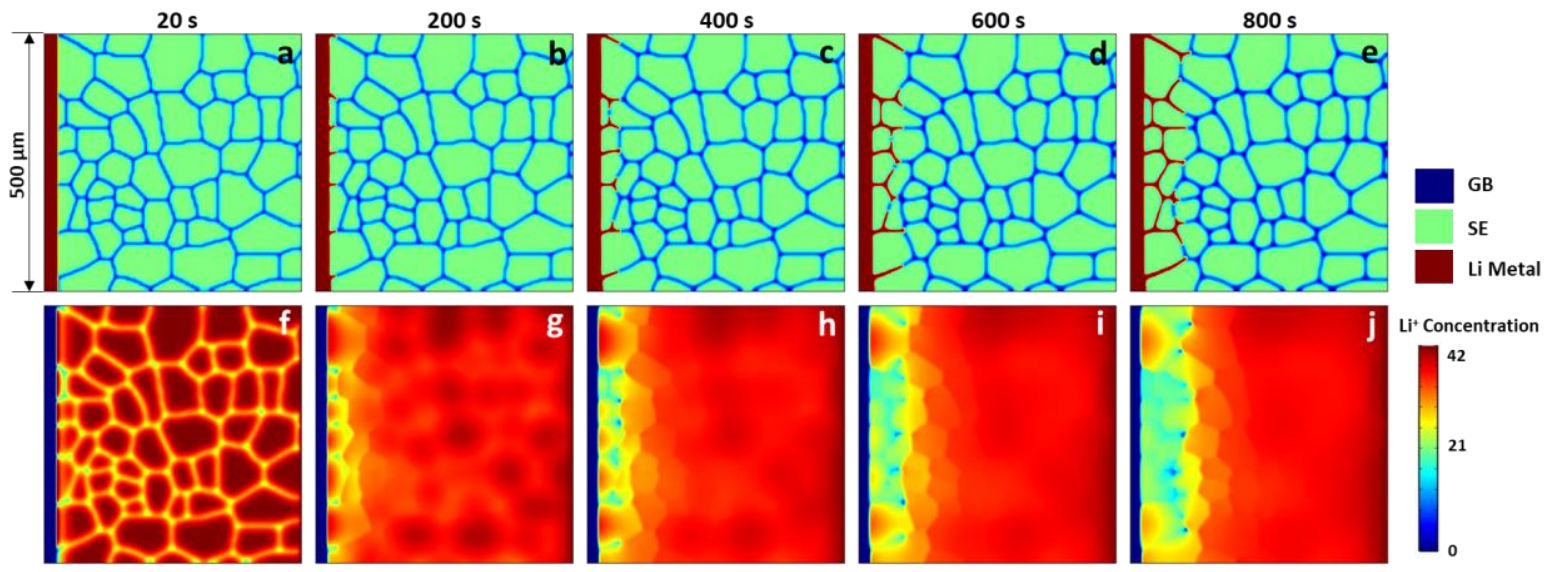

j $\mathrm{Li}^{+}$Concentration (M)
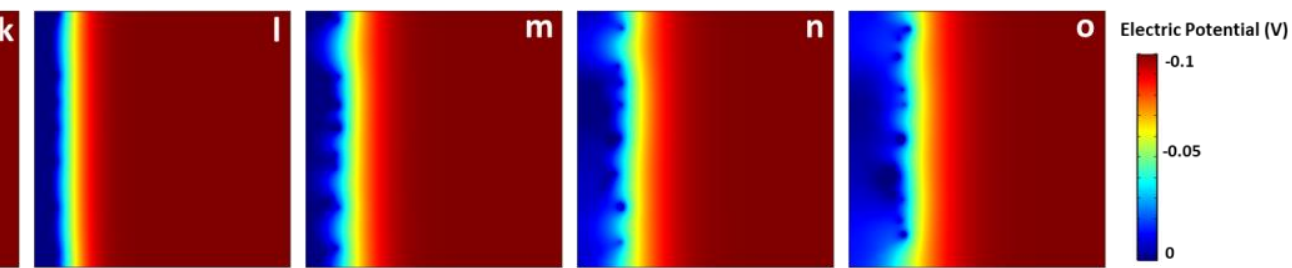

Figure S5. Morphology, Li ion concentration and electric potential evolution during electrodeposition in ideal LLZO SSEs of $75 \mu \mathrm{m}$ grain size with no surface electrons.
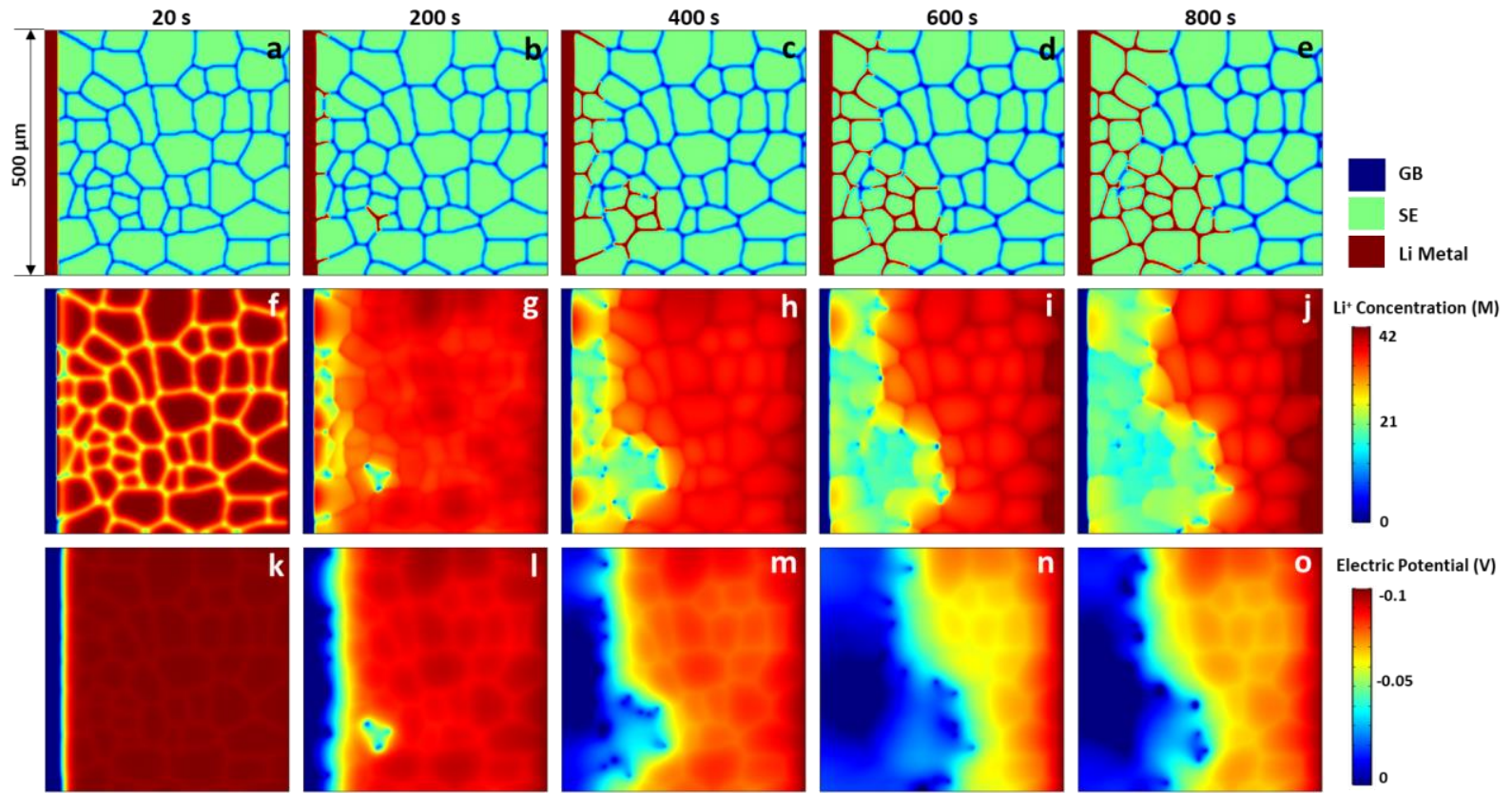

Figure S6. Morphology, Li ion concentration and electric potential evolution during electrodeposition in real LLZO SSEs of $75 \mu \mathrm{m}$ grain size with excess surface electrons. 


\section{References}

1. Rangasamy, E., J. Wolfenstine, and J. Sakamoto, The role of Al and Li concentration on the formation of cubic garnet solid electrolyte of nominal composition Li7La3Zr2012. Solid State Ionics, 2012. 206: p. 28-32.

2. Xie, H., et al., Lithium Distribution in Aluminum-Free Cubic Li7La3Zr2O12. Chemistry of Materials, 2011. 23(16): p. 3587-3589.

3. Cussen, E.J., The structure of lithium garnets: cation disorder and clustering in a new family of fast Li+ conductors. Chemical Communications, 2006(4): p. 412-413.

4. O'Callaghan, M.P. and E.J. Cussen, Lithium dimer formation in the Li-conducting garnets Li5+xBaxLa3-xTa2O12 (0<x<= 1.6). Chemical Communications, 2007(20): p. 2048-2050.

5. Tian, H.-K., B. Xu, and Y. Qi, Computational study of lithium nucleation tendency in Li 7 La 3 Zr 2 $O 12$ (LLZO) and rational design of interlayer materials to prevent lithium dendrites. Journal of Power Sources, 2018. 392: p. 79-86.

6. Homma, K., et al., Crystal structure and phase transitions of the lithium ionic conductor Li3PS4. Solid State lonics, 2011. 182(1): p. 53-58.

7. Lepley, N.D., N.A.W. Holzwarth, and Y.J.A. Du, Structures, Li+ mobilities, and interfacial properties of solid electrolytes Li3PS4 and Li3PO4 from first principles. Physical Review B, 2013. 88(10): p. 11.

8. Aono, H., et al., IONIC-CONDUCTIVITY OF THE LITHIUM TITANIUM PHOSPHATE (LI1+XALXTI2$X(P O 4) 3),(L I 1+X S C X T I 2-X(P O 4) 3),(L I 1+X Y X T I 2-X(P O 4) 3),(L I 1+X L A X T I 2-X(P O 4) 3$ SYSTEMS. Journal of the Electrochemical Society, 1989. 136(2): p. 590-591.

9. Dashjav, E. and F. Tietz, Neutron Diffraction Analysis of NASICON-type Li1+xAlxTi2-xP3O12. Zeitschrift für anorganische und allgemeine Chemie, 2014. 640(15): p. 3070-3073.

10. Redhammer, G., et al., A single crystal $X$-ray and powder neutron diffraction study on NASICON-

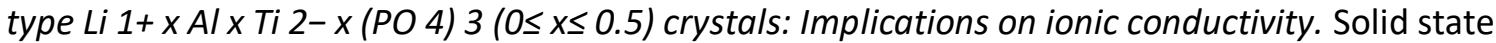
sciences, 2016. 60: p. 99-107.

11. Zhu, Y.Z., X.F. He, and Y.F. Mo, First principles study on electrochemical and chemical stability of solid electrolyte-electrode interfaces in all-solid-state Li-ion batteries. Journal of Materials Chemistry A, 2016. 4(9): p. 3253-3266.

12. Deng, Z., et al., Elastic Properties of Alkali Superionic Conductor Electrolytes from First Principles Calculations. Journal of the Electrochemical Society, 2016. 163(2): p. A67-A74.

13. Lang, B., B. Ziebarth, and C. Elsasser, Lithium Ion Conduction in LiTi2(PO4)(3) and Related Compounds Based on the NASICON Structure: A First-Principles Study. Chemistry of Materials, 2015. 27(14): p. 5040-5048.

14. Yang, J., et al., A High-Rate and Ultralong-Life Sodium-Ion Battery Based on NaTi2(PO4)(3) Nanocubes with Synergistic Coating of Carbon and Rutile TiO2. Small, 2015. 11(31): p. 37443749.

15. Pearse, A.J., et al., Nanoscale Solid State Batteries Enabled by Thermal Atomic Layer Deposition of a Lithium Polyphosphazene Solid State Electrolyte. Chemistry of Materials, 2017. 29(8): p. 3740-3753.

16. Senevirathne, K., et al., A new crystalline LiPON electrolyte: Synthesis, properties, and electronic structure. Solid State Ionics, 2013. 233: p. 95-101.

17. Holzwarth, N., First Principles Modeling of Electrolyte Materials in All-Solid-State Batteries. Physics Procedia, 2014. 57: p. 29-37.

18. Kresse, G. and J. Furthmüller, Efficient iterative schemes for ab initio total-energy calculations using a plane-wave basis set. Physical review B, 1996. 54(16): p. 11169. 
19. Liu, Z., et al., Anomalous high ionic conductivity of nanoporous B-Li3PS4. Journal of the American Chemical Society, 2013. 135(3): p. 975-978.

20. Perdew, J.P., K. Burke, and M. Ernzerhof, Generalized gradient approximation made simple. Physical review letters, 1996. 77(18): p. 3865.

21. Thompson, T., et al., Electrochemical Window of the Li-lon Solid Electrolyte Li7La3Zr2012. Acs Energy Letters, 2017. 2(2): p. 462-468.

22. Chen, L.J., et al., Oxygen vacancy in LiTiPO5 and LiTi2(PO4)(3): A first-principles study. Physics Letters A, 2011. 375(5): p. 934-938.

23. Kresse, G. and D. Joubert, From ultrasoft pseudopotentials to the projector augmented-wave method. Physical Review B, 1999. 59(3): p. 1758.

24. Heyd, J., G.E. Scuseria, and M. Ernzerhof, Hybrid functionals based on a screened Coulomb potential. The Journal of chemical physics, 2003. 118(18): p. 8207-8215.

25. Krukau, A.V., et al., Influence of the exchange screening parameter on the performance of screened hybrid functionals. The Journal of chemical physics, 2006. 125(22): p. 224106.

26. Chen, L., et al., Modulation of dendritic patterns during electrodeposition: A nonlinear phasefield model. Journal of Power Sources, 2015. 300: p. 376-385.

27. Fudong Han, A.S.W., Jie Yue, Xiulin Fan, Fei Wang, Miaofang Chi, Donovan N. Leonard, Nancy J. Dudney, Howard Wang, Chunsheng Wang High electronic conductivity as the origin of lithium dendrite formation within solid electrolytes. Nature energy, 2019.

28. Murugan, R., V. Thangadurai, and W. Weppner, Fast lithium ion conduction in garnet-type Li7La3Zr2012. Angewandte Chemie-International Edition, 2007. 46(41): p. 7778-7781. 